\title{
Moderne Wundbehandlung
}

A ufgrund der großen regenerativen Potenz unseres Organismus heilen Wunden in der Regel ohne ärztliches Zutun. Erst bei Ausbleiben der Heilung zeigt sich, wie komplex der Reparationsvorgang ist. Die Ursache ist oftmals Ausdruck tiefer liegender Probleme, die ihren Ursprung in der Wundentstehung und gesundheitlichen Verfassung des Patienten haben. Aus diesem Grund bedarf die Wundbehandlung mit Ausnahme von Bagatellwunden einer ärztlichen Versorgung in Form einer exakten diagnostischen Abklärung, gegebenenfalls chirurgischen Maßnahmen und einer strukturierten Weiterbehandlung.

Dabei stellt sich als Vorteil heraus, dass sich gerade im Bereich der Wundbehandlung eine zunehmende Spezialisierung einstellt. Gebietsübergreifende Kooperationen von Gefäßchirurgen, plastischen Chirurgen, Diabetologen, Angiologen, Dermatologen und Phlebologen haben das Outcome solcher komplexer Therapien deutlich verbessert. Noch liegt die Behandlung chronischer Wunden mit über 30\% in chirurgischer Hand, etwa $20 \%$ der Wunden werden von Hautärzten und $15 \%$ von Allgemeinmedizinern versorgt. Denkbar ist, dass sich die Wundversorgung weiter spezialisiert und etabliert und im Rahmen dieser Entwicklung eine eigenständige Fachdisziplin entsteht.

Ohne entsprechende Unterstützung der Pflegedienste wäre vor allem die ambulante Wundversorgung nicht möglich. Doch auch diese Berufsgruppe zeigt reges Interesse an dem Thema, und es existiert eine fundierte Weiterbildung, sodass sich inzwischen eine interdisziplinäre Zusammenarbeit zwischen Ärzten und Pflegedienst entwickelt - mit erfreulich guten Ergebnissen zum Wohle des Patienten.

Die nachfolgenden Beiträge sollen aus der Vielzahl von Veröffentlichungen und Themen bei der Behandlung von Wundproblemen einige spezielle Aspekte nochmals aufgreifen und diskutieren. Wegen der Produktvielfalt wurde darauf verzichtet, auf die verschiedensten Präparate detailliert einzugehen, die erwähnten Produkte stehen stellvertretend für alle anderen. Die Beiträge sollen eine Hilfe bei der Orientierung der rationellen Verfahrenswahl sein, gleichzeitig aber auch die Grenzen der lokalen Wundversorgung mit dem Blick auf eine ganzheitliche Therapie aufzeigen. Hervorzuheben ist nach wie vor die Tatsache, dass die Aufmerksamkeit weniger auf das pharmazeutische Produkt als vielmehr auf den Patienten und seine Wunde gelegt werden.
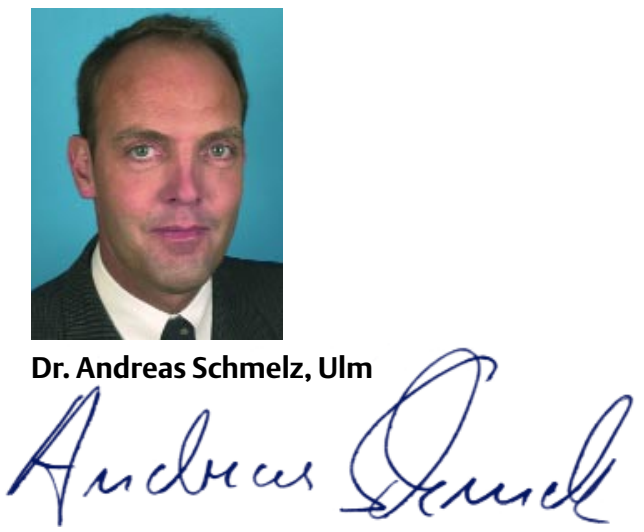